\title{
Extragalactic $\gamma$-ray propagation: beyond the absorption-only model
}

\author{
Timur Dzhatdoev ${ }^{a,+*}$, Emil Khalikov ${ }^{a}$ and Anna Kircheva ${ }^{a, b}$ \\ ${ }^{a}$ Federal State Budget Educational Institution of Higher Education M.V. Lomonosov Moscow \\ State University, Skobeltsyn Institute of Nuclear Physics (SINP MSU), 1(2), Leninskie gory, \\ GSP-1, Moscow 119991, Russian Federation \\ ${ }^{b}$ Federal State Budget Educational Institution of Higher Education, M.V. Lomonosov Moscow \\ State University, Department of Physics, 1(2), Leninskie gory, GSP-1, 119991 Moscow, Russian \\ Federation \\ ${ }^{+}$E-mail: timur1606@gmail.com
}

Observations of extragalactic sources in the high energy (HE, E $>100 \mathrm{MeV}$ ) and very high energy (VHE, E>100 GeV) domains allow to search for axion-like particles (ALP) and constrain the extragalactic magnetic field (EGMF) parameters. Such studies have recently received much attention thanks to the advent of new instruments and the progress of the extragalactic background light (EBL) models. Observations of blazars indicate that an additional component from electromagnetic (EM) cascades contributes to the observed flux, allowing for a possibility that the EGMF strength in voids of the large scale structure is smaller than $1 \mathrm{fG}$ on the characteristic spatial scale of $1 \mathrm{Mpc}$. We consider various extragalactic $\gamma$-ray propagation models with an emphasis on the "electromagnetic cascade model", which assumes that primary particles are $\gamma$-rays, and has the following spectral signatures: 1) a high-energy cutoff caused by the $\gamma \gamma \rightarrow e^{+} e^{-}$absorption process 2) an "ankle" formed by the intersection of the primary and cascade components 3) a possible low-energy "magnetic cutoff", 4) a possible low-energy "second ankle" at the second intersection of the primary and cascade components. The ankle may mimic the signature of the $\gamma$-ALP oscillation process. Finally, we consider various species of the "hadronic cascade model", in which primary particles are protons and nuclei. This model has a number of difficulties, but, nevertheless, is not excluded.

35th International Cosmic Ray Conference - ICRC2017

10-20 July, 2017

Bexco, Busan, Korea

* Speaker. 


\section{Introduction}

Extragalactic background light (EBL), that is, time-integrated radiation of all sources in the Universe, appears to be extremely difficult to measure directly due to strong foregrounds (e.g., [1]). Some measurements, such as [2], indicate the possible presence of an unaccounted EBL component(s) of whatever nature. The extragalactic magnetic field (EGMF) and other physical conditions in the intergalactic medium, such as the spatial maps of density and temperature, are also poorly known. Observations with $\gamma$-ray telescopes, such as Fermi LAT [3], H.E.S.S. [4], MAGIC [5], VERITAS [6], and future detectors such as the CTA array [7]-[8] and the GAMMA400 telescope [9], may allow to constrain a wide range of existing models intended to describe propagation of very high energy (VHE, $E>100 \mathrm{GeV}$ ) $\gamma$-rays and their secondary electrons and positrons (hereafter simply "electrons"). In this contribution we review the available models of extragalactic $\gamma$-ray propagation with an emphasis on the so-called "cascade models" which account for the cascade process that produces secondary photons on the cosmic microwave background $(\mathrm{CMB})$ and $\mathrm{EBL}$.

The paper is organized as follows. In Sect. 2 a classification scheme of $\gamma$-ray propagation models is presented. In Sect. 3 we discuss the general principles that may be used to select the "best" model. Sect. 4 discusses the electromagnetic cascade model, and Sect. 5 - the hadronic cascade model. Finally, we draw our conclusions in Sect. 6.

\section{Classification of extragalactic $\gamma$-ray propagation models}

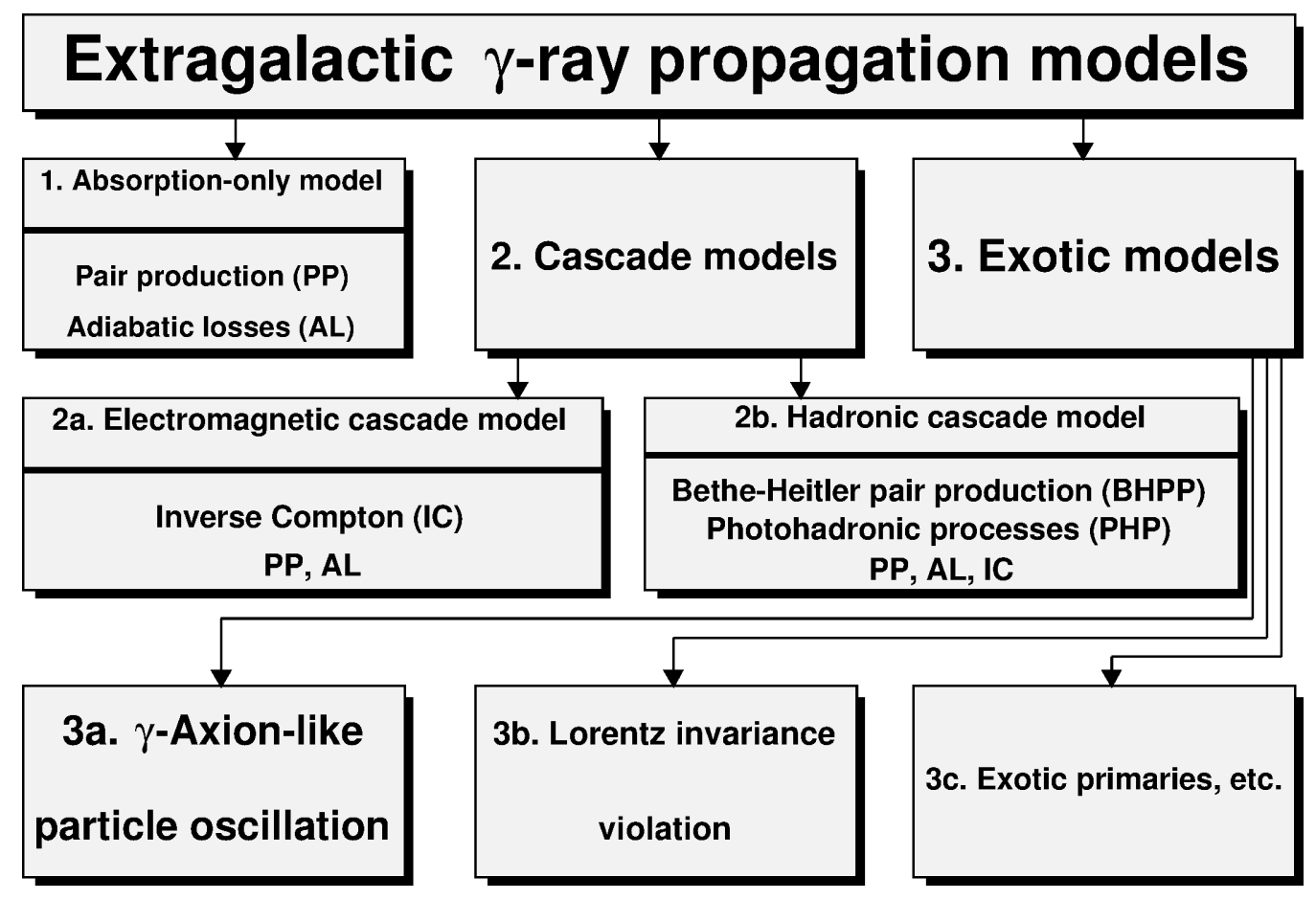

Figure 1: Classification of extragalactic $\gamma$-ray propagation models proposed in the present work. 
Here we adopt a classification scheme of existing extragalactic $\gamma$-ray propagation models (see Fig. 1) that is very similar to the one proposed in [10]. There are three broad classes of these models:

1. Currently the most popular is the so-called "absorption-only model", that accounts for only $\gamma \gamma \rightarrow e^{+} e^{-}$pair production (PP) by primary photons and their adiabatic losses (AL). The first works that discussed $\gamma \gamma$ absorption were [11]-[12]; for a recent brief review see [10].

2. Energy of primary $\gamma$-rays does not disappear after PP interactions, but is rather being transferred to secondary electrons. These electrons, in turn, may produce inverse Compton (IC) secondary (cascade) $\gamma$-rays. EGMF strength in voids of the large scale structure is still poorly constrained [13]-[14] (for a recent compilation of EGMF constraints see [15]). Therefore, the possibility that cascade electron deflection angles are small and secondary $\gamma$-rays contribute to the image of a point-like extragalactic source can not be excluded at present (however, AGN outflows may produce rather strong EGMF [16]-[17]; in this case cascade electrons with an energy $E<1 \mathrm{TeV}$ may be practically isotropized and, consequently, the contribution of the cascade component of $\gamma$-rays to observable spectra of beamed sources like blazars almost suppressed). Intergalactic cascade models may be further divided to two types by the nature of the primary particle (of course, the third type of both primaries at the same time may be considered as well):

2a. The electromagnetic cascade model (ECM) assumes that all observable emission comes from primary $\gamma$-rays. The simplest physically motivated ECM postulates primary (intrinsic) spectrum $\propto E^{-\gamma} \exp \left(-E / E_{c}\right)$; therefore, it predicts two observable components: a comparatively hard component of primary $\gamma$-rays that did not undergo absorption on the EBL photons (see, e.g., [18], Fig. 12, top-right, solid black curve) and a comparatively soft cascade component (the same figure, green curve). A more complex shape of the primary spectrum is also imaginable; for example, the source may emit an additional component of photons with superhigh energy (SHE, E $>100 \mathrm{TeV}$ ) presumably by a hadronic mechanism [19]. Provided that the distance to the source is large enough (redshift $z>0.02$ already satisfies this condition, see [18], Appendix A), these multi- $\mathrm{TeV} \gamma$-rays are reprocessed to cascade photons in the $\mathrm{GeV}-\mathrm{TeV}$ energy range with a certain ("universal") spectrum that is practically independent on the primary spectrum in the SHE range, but may strongly depend on $z$ ("weak universality", see [20]). This case of primary SHE cascade $\gamma$-rays was considered in [10]; it is interesting in connection with the ongoing search for the sources of IceCube astrophysical neutrinos [21] that accompany primary SHE "hadronic" $\gamma$-rays [22],[23].

2b. The hadronic cascade model (HCM) [24]-[26] (for more references see [10], [18]) includes a cascade component from SHE and ultra-high energy (UHE, $E>1 \mathrm{EeV}$ ) $\gamma$-rays and electrons, which, in turn, were produced by a beam of ultra-high energy cosmic rays (CR) by means of Bethe-Heitler pair production (BHPP) $N \gamma \rightarrow N^{\prime} e^{+} e^{-}$or photohadronic processes (PHP) $N \gamma \rightarrow$ $N^{\prime}\left(n_{1}\right) \pi^{+}\left(n_{2}\right) \pi^{-}\left(n_{3}\right) \pi^{0}$ with subsequent decays of pions (here $N$ denotes a proton or nucleus, each number $\left(n_{1}, n_{2}, n_{3}\right)$ may take value $\left.0,1,2, \ldots\right)$. In [18] we suggested a further sub-division to distinguish "basic", "intermediate", and "modified" species of the HCM. The basic HCM [27] postulates that all observable $\gamma$-rays were produced by CR in the intergalactic space. The intermediate HCM, first considered in [18], includes the possibility that the beam is dissipated (i.e. CR are deflected to large angles $>>0.1^{\circ}$ ) at some $z=z_{c}$ by strong local magnetic fields on the way from the source to the observer. Finally, the "modified" HCM [28] allows an additional contribution from primary $\gamma$-rays that survived absorption on the EBL. 
3. As well, there exist some models that postulate effects of new physics, such as $\gamma$-axion-like particle (ALP) mixing [29] (for recent treatment of this effect see [30]-[31]; a very recent work on laboratory axion-like particle search [32]), Lorentz invariance violation (LIV), or exotic primaries (for more references see [10], [18]). These models were mainly devised in order to explain the apparent excess of intensity at the highest-energy bins in observed spectra of some blazars that from time to time was claimed by several groups (e.g., [33]-[35]).

\section{What model to choose?}

By now, observation of blazars revealed several effects that go beyond the absorption-only model (in what follows we call these deviations "anomalies", even if their explanation does not call for any new physics); a short review of these anomalies may be found in [18]; the corresponding works are mentioned here in Sect. 2 and Sect. 4. If the absorption-only model falls short of the universal $\gamma$-ray propagation model for blazars, it is natural that some other model should replace it. What model, then, should be preferred? Below we advocate the use of several principles instructive to settle this important question.

1. The simplest model that would be able to explain all observed effects is preferable ("the Occam's razor").

2. Nevertheless, it is good not to put too much emphasis on any particular anomaly. Therefore, we formulate the second rule: consider every observed anomaly, not the odd anomaly.

3. Sometimes a particular model provides a prediction (e.g., for the observable spectrum of a source) that is in striking coincidence with observations. Nevertheless, we argue against putting too much faith in such coincidences, especially if the assumptions of this model are not in harmony with other well established pieces of astrophysical knowledge. In other words: better (astro)physically motivated models should have a priority.

4. Finally, it is good not to reject any model only on grounds of "common sense" that is not supported by any measurement. For instance, cascade models from beamed extragalactic sources for several decades were considered unworthy precisely because the EGMF strength "can not" be as low as $1 f G$ or lower. An unprejudiced examination, however, fails to discoved any compelling reason for this belief.

\section{EM cascade model and its signatures}

Let us examine, then, the electromagnetic cascade model that would be clearly preferred by the Occam's principle. Indeed, only two assumptions are necessary for the cascade component to contribute significantly to the observable spectrum: 1) the EGMF strength is low enough so that cascade electrons are deflected to small angles, typically less than $1^{\circ} 2$ ) the primary spectrum is hard enough, allowing the cascade component dominance at low energy. The first assumption at present remains conjectural, but is not excluded by any firm measurement, and is even favoured by [36]-[37]. The second assumption is justified by the results of [38].

By now, at least seven observable signatures of the ECM were discussed in the literature, namely: 
1. Spectral signatures [39]:

1a. High-energy cutoff due to $\gamma \gamma$ absorption

1b. Ankle at the intersection of the attenuated primary and the cascade component. This signature may explain the high-energy anomaly of [34]-[35] (see [18] for a detailed discussion)

1c. Magnetic cutoff [10] (see figure 5, left)

$1 \mathrm{~d}$. Second ankle at the low-energy intersection of the attenuated primary and the cascade component [39] (for a tentative observation of such a break see [38])

2. Angular signatures, such as the magnetically broadened cascade (MBC) [40] and/or the pair halo $(\mathrm{PH})$ patterns [19] ([36] obtained an indication for such a pattern)

3. Timing signatures, such as the energy-dependent delay [41]-[43],[14]

4. Signatures of a sample of objects, such as the voidiness-dependent effect of [37].

In effect, all known anomalies find their natural explanation in the framework of the ECM. Up to our knowledge, at present there is no evidence against the ECM from any astrophysical measurement. Therefore, we conclude that this model fulfills all four of our criteria formulated in Sect. 3.

\section{Hadronic cascade model and its difficulties}

The HCM is founded on the following assumptions: 1) the EGMF strength is low enough so that electrons in electromagnetic cascades are deflected to small angles (this one is the same assumption as for the case of the ECM) 2) the beamed source accelerates CR (protons or nuclei) up to the energy of $10 \mathrm{EeV}$ or more (this assumption is needed to ensure that a substantial part of the CR energy is transferred to observable $\gamma$-rays) 3) the CR luminosity of the blazar is high enough to ensure a substantial contribution of cascade $\gamma$-rays to the observable spectrum and/or 4) $\mathrm{CR}$ accelerated by the blazar are strongly beamed and magnetic field around the blazar is low enough so that accelerated $\mathrm{CR}$ are deflected to small angles $\left(<5^{\circ}\right)$. From the very start, it is evident that the HCM requires more assumptions than the ECM. Therefore, at present the ECM should be preferred. Let us see how the HCM meets other requirements listed in Sect. 3.

In [18] we presented a detailed calculations of observable spectra in the framework of the HCM together with a painstaking account of the calculation methods used by us, and limitations of these methods. In particular, two different approaches to the calculation of observable spectra were devised to ensure robustness of results, namely: 1) assuming "weak universality" and 2) without such an assumption. As well, our calculations were done assuming contemporary, realistic EBL model of [44].

For the case of the basic HCM, we found a new region of an almost strictly universal shape above $20 \mathrm{TeV}$ and explained it qualitatively (see figure 7). However, the basic HCM seems to have a limited range of applicability when fitting the observed spectra of blazars. It seems to be insufficient to describe the spectra of some blazars considered in [18], but the intermediate HCM usually fits them well if the $z_{c}$ parameter is left free. As well, we provided strong constraints on the basic and intermediate HCM for the case of 1ES 0229+200, assuming the blazar emission model of [45], and the model of the surrounding turbulent magnetic field from [46]. It turns out that any realistic model under these assumptions is excluded with statistical significance $Z>7 \sigma$. Therefore, 
these two models appear to be astrophysically implausible for some blazars (i.e. our criterion 3 in not fulfilled for them).

Finally, we considered the case of the modified HCM. As a rule, for the case of assumptions examined in [18] we could obtain good fits for observed spectra, but in this case the attenuated component usually dominates at low energy $E<300 \mathrm{GeV}$, thus making it difficult to accomodate for the effect claimed in [36], and almost impossible to explain the ones discussed in [37]. Therefore, the modified HCM fails to meet our criterion 2. To conclude, any unprejudiced analysis clearly favors the ECM.

\section{Conclusions}

There is rather solid (and mounting) evidence that the absorption-only model is incomplete. The electromagnetic cascade model may, in principle, explain all these deviations (anomalies). The most basic versions of hadronic cascade model are excluded; other options of this model are not favoured. Finally, the option of the extragalactic magnetic field's strength of $1 a G-1 f G$ on the 1 $M p c$ scale still remains viable.

\section{References}

[1] E. Dwek \& F. Krennrich, The extragalactic background light and the gamma-ray opacity of the universe, APh, 43 (2013) 112 [astro-ph/1209.4661].

[2] S. Matsuura et al., New Spectral Evidence of an Unaccounted Component of the Near-infrared Extragalactic Background Light from the CIBER, ApJ, 839 (2017) 7 [astro-ph/1704 07166 ].

[3] W.B. Atwood et al, The Large Area Telescope on the Fermi Gamma-ray Space Telescope Mission, ApJ, 697 (2009) 1071 [astro-ph/0902.1089]

[4] J.A. Hinton, The status of the HESS project, New Astron. Rev., 48 (2004) 331 [astro-ph/0403052]

[5] E. Lorenz, Status of the 17 m diameter MAGIC telescope, New Astron. Rev., 48 (2004) 339

[6] F. Krennrich et al., VERITAS: the Very Energetic Radiation Imaging Telescope Array System, New Astron. Rev., 48 (2004) 345 [astro-ph/ 0108478 ]

[7] M. Actis et al., Design concepts for the Cherenkov Telescope Array CTA: an advanced facility for ground-based high-energy gamma-ray astronomy, ExA, 32 (2011) 193 [astro-ph/1008 . 3703]

[8] B.S. Acharya et al., Introducing the CTA Concept, APh, 43 (2013) 3

[9] A.M. Galper et al., Design and Performance of the GAMMA-400 Gamma-Ray Telescope for the Dark Matter Searches, AIP Conference Proceedings, 1516 (2013), 288 [astro-ph/1210 . 1457 ]

[10] T.A. Dzhatdoev, The intergalactic electromagnetic cascade solution for the anomalies from $\gamma$-ray blazar observations (2017) [ast ro-ph/1705.05360]

[11] A.I. Nikishov, Absorption of high-energy photons in the Universe, Sov. Phys. JETP 14 (1962) 393

[12] R.G. Gould \& G.P. Shreder, Opacity of the Universe to high-energy photons, PRL 16 (1966), 252

[13] A. Neronov \& Ie. Vovk, Evidence for Strong Extragalactic Magnetic Fields from Fermi Observations of TeV Blazars, Science, 328 (2010) 73 [astro-ph/1006.3504] 
[14] C.D. Dermer et al., Time delay of cascade radiation for TeV blazars and the measurement of the intergalactic magnetic field, ApJ Lett., 733 (2011) L21 [astro-ph/1011. 6660]

[15] T.A. Dzhatdoev el al., Testing models of extragalactic $\gamma$-ray propagation using observations of extreme blazars in GeV and TeV energy ranges, J. Phys.: Conf. Ser., 798 (2017) 012002 [astro-ph/1701.06827]

[16] S.R. Furlanetto \& A. Loeb, Intergalactic Magnetic Fields from Quasar Outflows, ApJ, 556 (2001) 619 [astro-ph/0102076]

[17] A.M. Beck et al., On the magnetic fields in voids, MNRAS Lett., 429 (2013) L60 [astro-ph/1210.8360]

[18] T.A. Dzhatdoev et al., Electromagnetic cascade masquerade: a way to mimic $\gamma$-axion-like particle mixing effects in blazar spectra, A\&A, 603 (2017) A59 [astro-ph/1609.01013]

[19] F.A. Aharonian et al., Very high energy gamma rays from active galactic nuclei: cascading on the cosmic background radiation fields and the formation of pair halos, ApJ, 423 (1994) L5-L8

[20] V. Berezinsky \& O. Kalashev, High-energy electromagnetic cascades in extragalactic space: Physics and features, Phys.Rev.D, 94 (2016) 023007 [astro-ph/1603.03989]

[21] M.G. Aartsen et.al., Evidence for High-Energy Extraterrestrial Neutrinos at the IceCube Detector, Science, 342 (2013) 1242856

[22] V.S. Berezinsky, A.Iu. Smirnov, Cosmic neutrinos of ultra-high energies and detection possibility, Ap\&SS, 32 (1975) 461

[23] V.S. Berezinsky et al., Restricting UHECRs and cosmogenic neutrinos with Fermi-LAT, Phys.Lett.B, 695 (2011) 13 [astro-ph/1003.1496]

[24] A. Uryson, Possible observation of electromagnetic cascades in extragalactic space, Sov.Phys.JETP, 113 (1998) 385

[25] W. Essey, A. Kusenko, A new interpretation of the gamma-ray observations of distant active galactic nuclei, APh, 33 (2010) 81 [astro-ph/0905.1162]

[26] K. Murase et al., Blazars as Ultra-High-Energy Cosmic-Ray Sources: Implications for TeV Gamma-Ray Observations, ApJ, 749 (2012) 63 [astro-ph/1107.5576]

[27] W. Essey et al., Secondary Photons and Neutrinos from Cosmic Rays Produced by Distant Blazars, Phys.Rev.Lett., 104 (2010) 141102 [astro-ph/ 0912 .3976]

[28] W. Essey \& A. Kusenko, Understanding the spectrum of a distant blazar PKS 1424+240 and its implications, APh, 57 (2014) 30 [astro-ph/1310.3440]

[29] G. Raffelt \& L. Stodolsky, Mixing of the photon with low-mass particles, Phys. Rev. D, 37 (1988) 1237

[30] A. Kartavtsev et al., Extragalactic photon-ALP conversion at CTA energies, JCAP, 01 (2017) 024 [astro-ph/1611.04526]

[31] D. Montanino et al., Enhancing the spectral hardening of cosmic TeV photons by the mixing with axion-like particles in the magnetised cosmic web (2017) [astro-ph/1703.07314]

[32] V. Anastassopoulos et al., Towards a medium-scale axion helioscope and haloscope (2017) [astro-ph/1706.09378]

[33] R.J. Protheroe \& H. Meyer, An infrared background-TeV gamma-ray crisis?, Phys.Lett.B, 493 (2000) 1 [astro-ph/0005349] 
[34] D. Horns, M. Meyer, Indications for a pair-production anomaly from the propagation of VHE gamma-rays, JCAP, 02 (2012) 033 [astro-ph/1201 . 4711]

[35] G.I. Rubtsov \& S.V. Troitsky, Breaks in gamma-ray spectra of distant blazars and transparency of the Universe, JETP Letters, 100 (2014) 355 [astro-ph/1406 . 0239]

[36] W. Chen et al., Search for GeV $\gamma$-Ray Pair Halos Around Low Redshift Blazars, Phys.Rev.Lett., 115 (2015) 211103 [astro-ph/1410. 7717]

[37] A. Furniss et. al., A correlation between hard gamma-ray sources and cosmic voids along the line of sight, MNRAS, 446 (2015) 2267 [astro-ph/1407.6370]

[38] A. Neronov, et. al., Very hard gamma-ray emission from a flare of Mrk 501, A\&A 541 (2012) A31 [astro-ph/1104.2801]

[39] T.A. Dzhatdoev et al., The VHE anomaly in blazar spectra and related phenomen, Bull. Rus. Acad. Sci., 81 (2017) 4, 443 [astro-ph/1701.07293]

[40] A. Abramowski et al., Search for extended $\gamma$-ray emission around AGN with H.E.S.S. and Fermi-LAT, A\&A 562 (2014) A145 [astro-ph/1401.2915]

[41] R. Plaga, Detecting intergalactic magnetic using time delays in pulses of $\gamma$-rays, Nature, 374 (1995) 430

[42] K. Ichiki et al., Probing the Nature of the Weakest Intergalactic Magnetic Fields with the High-Energy Emission of Gamma-Ray Bursts, ApJ, 682 (2008) 127 [astro-ph/ 0711.1589 ]

[43] K. Murase et al., Probing Intergalactic Magnetic Fields in the GLAST Era through Pair Echo Emission from TeV Blazars, ApJL 686 (2008) L67 [astro-ph / 0806 . 2829]

[44] T.M. Kneiske \& H. Dole, A Lower-Limit Flux for the Extragalactic Background Light Astronomy and Astrophysics, A\&A, 515 (2010) A19 [astro-ph/1001.2132]

[45] F. Tavecchio, On the hadronic cascade scenario for extreme BL Lacs, MNRAS, 438 (2014) 3255 [astro-ph/1312.4281]

[46] M. Meyer, et al., First lower limits on the photon-axion-like particle coupling from very high energy gamma-ray observations, Phys.Rev.D, 87 (2013) 035027 [astro-ph/1302 .1208] 